\title{
Antisense Oligonucleotide Gene Therapy for Neuromuscular Disorders
}

\author{
Ryan Gotesman \\ Faculty of Medicine, University of Ottawa, Ontario, Canada
}

ABSTRACT

Antisense oligonucleotides (ASOs) are synthetic, single-stranded DNA molecules that can bind to specific mRNA sequences and alter protein expression. ASO gene therapies are leading to breakthroughs in the treatment of once intractable neuromuscular disorders. In 2016, ASOs became the first FDA-approved drugs for treating spinal muscular atrophy and Duchenne muscular dystrophy. Recent trials also suggest ASOs may be effective in combating Huntington's disease, amyotrophic lateral sclerosis and hereditary transthyretin amyloidosis. This article highlights ASOs' mechanism of action, their use in treating neuromuscular disease and future obstacles the gene therapy must overcome, providing an update on the state of ASO technology.

RÉSUMÉ

Les oligonucléotides anti-sens (ASO) sont des molécules synthétiques de l'ADN à simple brin, qui peuvent se lier à une séquence spécifique de l'ARNm et altérer l'expression des protéines. La thérapie génique par ASO mène des percées importantes dans le traitement des désordres neuromusculaires antérieurement intraitables. En 2016, les ASO sont devenues les premières drogues approuvées par la FDA pour le traitement de l'amyotrophie spinale et la myopathie de Duchenne. Des essais récents suggèrent que les ASO pourraient également être efficaces dans le traitement de la maladie de Huntington, de la sclérose latérale amyotrophique et de l'amylose de la transthyrétine héréditaire. Cet article souligne le mécanisme d'action des ASO, leurs usages dans le traitement des maladies neuromusculaires, ainsi que les obstacles futurs que la thérapie génique doit surmonter, donnant une mise à jour sur l'état de la technologie des ASO.

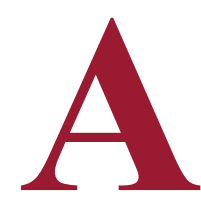

ntisense oligonucleotides (ASOs) are an emerging form of gene therapy that promise to transform the treatment of neuromuscular disorders. ASOs are synthetic, single-stranded DNA molecules, usually no more than 30 nucleotides long, that utilize the complementary nature of nucleotide base pairing to hybridize with a specific mRNA sequence and alter protein expression (1). The first antisense drug was approved by the US Food and Drug Administration (FDA) in 1998 to treat cytomegalovirus retinitis and ASOs have also been developed to combat familial hypercholesterolemia and age-related macular degeneration (2). ASOs significantly slowed the progression of each of these diseases and researchers have been eager to replicate this success in other disorders (2). Perhaps most exciting has been the success of ASOs in treating previously intractable neuromuscular illnesses. In 2016 the FDA approved two antisense therapies for spinal muscular atrophy and Duchenne muscular dystrophy (2). In addition, ongoing clinical trials are testing ASOs in Huntington's disease, amyotrophic lateral sclerosis, and other neurological disorders. With the recent completion of a trial demonstrating an ASO's efficacy in treating hereditary transthyretin amyloidosis, this article provides a timely update on how antisense drugs are quickly becoming an essential component in the neurologist's toolkit.

\section{MECHANISM OF ACTION}

The two primary mechanisms by which ASOs can impact protein expression are by targeting an mRNA transcript for degradation or by altering how the transcript is processed (3). The hybridization of an ASO with mRNA to form a DNARNA complex recruits the ribonuclease $H$ (RNase $H$ ) enzyme to hydrolyze the RNA strand (3). An advantage of these ASOs is that cleavage of the mRNA and downregulation of protein expression will occur regardless of the segment of mRNA that is targeted (Figure 1A). In contrast, ASOs that cannot recruit RNase $\mathrm{H}$, but instead alter aspects of RNA processing, must be carefully targeted to specific mRNA segments to elicit their effects. Binding at the start codon can inhibit ribosomal assembly (Figure 1B), preventing mRNA translation. Alternatively, the association with intron-exon junctions can stimulate the inclusion or exclusion of introns and exons (Figure 1C) and form novel protein variants with altered functionality $(4,5)$. Whether an ASO works through RNase $\mathrm{H}$ mediated cleavage or by influencing transcript processing depends on the chemical backbone of the drug

Keywords: Antisense oligonucleotides; ASO; Neuromuscular disorders; Gene therapy 


\section{APPLICATIONS IN DISEASE}

Spinal muscular atrophy

Spinal muscular atrophy (SMA) is a neuromuscular disorder caused by mutations in the SMN1 gene (6). SMA presents with degeneration of motor neurons, muscle atrophy and weakness. In infantile-onset, SMA typically leads to severe weakness by 6 months and respiratory failure by 2 years (7). The SMN1 gene encodes survival motor neuron (SMN), a protein necessary for motor neuron health, and mutations reduce the levels of functional SMN. The SMN2 gene can also code for SMN but due to alternative splicing, most of the gene's mRNA transcripts are lacking exon 7 and instead produce a non-functional SMN variant that is rapidly degraded (8).

Rather than attempt to treat the SMN1 mutation, researchers ingeniously targeted the alternative splicing of SMN2 using the ASO nusinersen. Nusinersen binds to a downstream intron of exon 7, modulating the splicing of SMN2's mRNA to include exon 7 and produce functional SMN (9). In a 13-month doubleblind randomized controlled trial, nusinersen was intrathecally administered to 121 infants with SMA (10). The nusinersen arm of the trial had a $47 \%$ lower risk of death or use of permanent assisted ventilation, compared to the control group. In addition, $51 \%$ of the infants receiving therapy were able to reach motor milestones such as sitting upright without aid, standing and rolling. However, no infants in the control group achieved these milestones. These results led to nusinersen receiving FDA approval in late 2016 and Health Canada approval in 2017, making this ASO the first treatment capable of slowing the progression of SMA.

Duchenne muscular dystrophy

Duchenne muscular dystrophy (DMD) is an X-linked neuromuscular disorder that leads to the breakdown and weakness of muscle (11). DMD is caused by a mutation in the gene encoding dystrophin, a structural protein that holds the cytoskeleton and plasma membrane together. Absence or dysfunction of this protein compromises muscle stability. DMD patients usually lose the ability to walk by age 12 and experience respiratory failure in their early twenties (11). About $13 \%$ of DMD cases are specifically caused by a nonsense mutation in exon 51 where an inappropriately placed stop codon produces a truncated, dysfunctional form of dystrophin (12).

The ASO eteplirsen binds to exon 51 and stimulates its excision, allowing for production of functional, albeit slightly shortened, dystrophin (13). In a double-blind placebo-controlled trial involving 12 patients, eteplirsen was intravenously infused for
48 weeks after which muscle biopsies were taken (14). Eteplirsen increased the percentage of dystrophin-positive fibers to $52 \%$ of normal. The drug also allowed DMD patients to walk an additional 67 meters, compared to placebo, in a 6-minute walk test. Despite these modest improvements, the FDA review team recommended against approving eteplirsen, citing the small sample size and questioning whether dystrophin levels had been enhanced enough to observe a true clinical benefit (15). Controversially, the FDA's director of the Center for Drug Evaluation and Research overruled the review team and granted eteplirsen accelerated approval, making it the first drug approved in the United States to treat DMD. A larger phase III trial involving about 110 patients is currently underway and a clearer answer on eteplirsen's efficacy is expected in 2020 (16).

\section{Huntington's disease}

Huntington's disease (HD) is a neurodegenerative disorder caused by expansion mutations in the huntingtin gene (17). The mutation stimulates the huntingtin protein to accumulate in toxic aggregates that induce degeneration of the striatum along with progressive motor deficits and cognitive dysfunction. Many in the HD community suspect the progression of the disease may be slowed by lowering levels of the mutant huntingtin protein. Indeed, genetic mutations that naturally reduce the expression of mutant huntingtin can delay disease onset by as much as 9.3 years (18). There is currently no treatment for HD though ASO technology may soon lead to a breakthrough. In a phase I/II trial, the ASO IONIS-HTTRX was intrathecally administered over 13 weeks to $46 \mathrm{HD}$ patients (19). Unlike nusinersen and eteplirsen, IONIS-HTTRX alters protein expression through RNase $\mathrm{H}$ mediated cleavage (20). After 3 months, there was a $40 \%-60 \%$ reduction of mutant huntingtin in the cerebrospinal fluid (21). Most importantly, no serious adverse events were reported, with none of the participants dropping out of the study. A phase II trial to further assess the safety and tolerability of IONIS-HTTRX in treating HD is ongoing (22).

Amyotrophic lateral sclerosis

Brought to prominence by Stephen Hawking, amyotrophic lateral sclerosis (ALS) is the most common form of motor neuron disease (23). ALS is caused by progressive degeneration of motor neurons, leading to muscle wasting, weakness and ultimately respiratory failure (23). Life expectancy after disease onset is only 3 to 5 years and current therapies have poor efficacy. Most ALS cases are sporadic, with no known cause. However, about $20 \%$ of familial ALS cases are caused by a mutation in the superoxide dismutase 1 (SOD1) gene (23). 


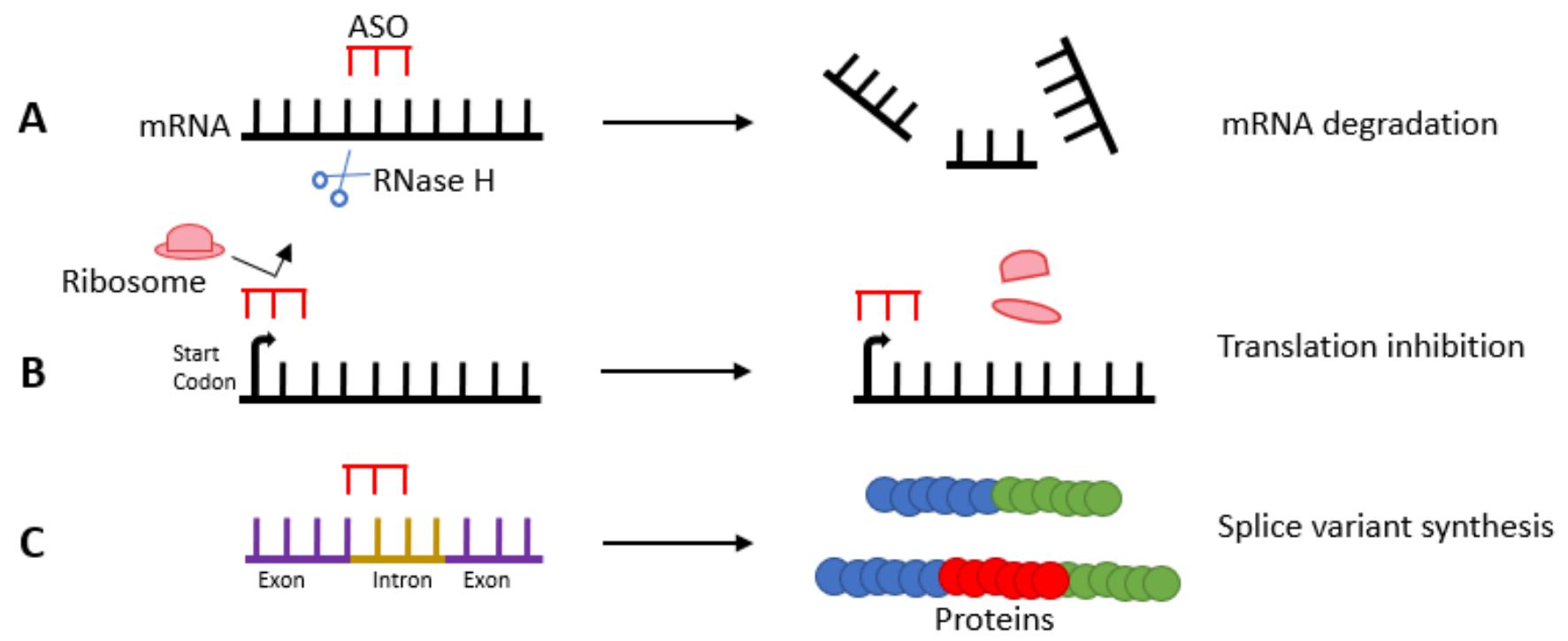

Figure 1: Antisense oligonucleotide (ASO) mechanisms of action - ASOs can impact protein expression by stimulating mRNA degradation or altering how the transcript is processed. (A) Binding between an ASO and mRNA can stimulate ribonuclease $\mathrm{H}$ (RNase $\mathrm{H}$ ) to cleave the mRNA, reducing protein expression. (B) An ASO can also bind at the start codon, preventing ribosomal assembly and lowering protein expression. (C) ASOs can also impact splicing of an mRNA transcript by binding at an intron-exon junction, altering the protein produced through translation.

SOD1 is an antioxidant enzyme that, when mutated, misfolds into toxic aggregates within motor neurons. There is hope that ASOs, which lower SOD1 levels may improve the disease. The feasibility of this approach was investigated in a phase I study where 32 patients with SOD1-positive ALS received intrathecal infusions of the ASO IONIS-SOD1Rx (24). Despite the ASO concentrations being too low to impact SOD1, no serious adverse events were reported. Another phase I trial investigating the safety and tolerability of the ASO is underway (25).

Hereditary transthyretin amyloidosis

Hereditary transthyretin amyloidosis (hATTR) is an autosomal dominant polyneuropathy caused by mutations in the transthyretin (TTR) encoding gene (26). Upon mutation, TTR misfolds to form insoluble amyloid fibrils that accumulate and impair various organ systems. Neurons are particularly impacted, and patients typically present with motor deficits alongside sensory and autonomic dysfunction (26). As the disease progresses, walking becomes increasingly difficult until a wheelchair is required. The average life expectancy is a mere 10 years after onset of symptoms. Since over $95 \%$ of mutant TTR is synthesized in the liver, liver transplantation is the standard of care though nerve function rarely improves after the surgery (26).
The ASO inotersen offers a far less invasive means of combating hATTR. Inotersen binds with TTR's mRNA transcript, stimulating cleavage by RNase $\mathrm{H}$ and reduction of protein levels (27). The ASO was tested in a double-blind, randomized, placebocontrolled phase III trial, where 172 hATTR patients received weekly subcutaneous injections of inotersen over a 15 month period (28). Average levels of TTR fell by $74 \%$ and the rate of neuropathy progression slowed within the inotersen group. Remarkably some patients even reported reversal of their neuropathy symptoms (28). Unfortunately, there were several serious adverse events with $54 \%$ of patients in the inotersen group experiencing thrombocytopenia and one patient dying from an intracranial haemorrhage. However, no serious adverse events occurred once enhanced monitoring was implemented (28). These results culminated with inotersen receiving drug approval within the European Union in July of 2018. The drug is currently under review by the FDA and Health Canada.

\section{FUTURE OBSTACLES}

A major drawback of ASO therapies is their exorbitant price. For instance, nusinersen costs $\$ 125000$ per injection (29). With the Canadian Agency for Drugs and Technology in Health recommending that health insurance only cover nusinersen for patients not requiring permanent invasive ventilation, it is pivotal that ASO therapies become less expensive (30). 
Another limitation of ASOs is their poor blood-brain barrier permeability (31). Whenever the central nervous system needs to be targeted, as in the case of nusinersen and IONIS-HTTRX, intrathecal injections are necessary and this can serve as a deterrent to starting therapy. Efforts are underway to enhance ASO blood-brain barrier permeability by encapsulating the ASO in extracellular vesicles or by tagging them with cellpenetrating peptides (32). These techniques also serve to improve the bioavailability of the drug. Finally, adverse reactions are a concern for any new class of drug and though nusinersen and eteplirsen appear safe in trials, inoserten induced serious adverse events. In addition, there is always the possibility of unintended off-target genetic effects.

\section{CONCLUSION}

ASOs are revolutionizing the management of SMA and DMD, and commit to do the same for HD, ALS and hATTR. By altering the expression of genes directly implicated in disease, ASOs can target the cause of an illness far more successfully than conventional drugs. They represent a promising new frontier in genetic therapy for helping patients with previously untreatable conditions. However, challenges remain particularly in the form of excessive prices, poor blood-brain barrier permeability and coping with adverse reactions. As the technology continues to advance we can expect many more exciting breakthroughs in the treatment of neuromuscular diseases.

\section{REFERENCES}

1. Dias N, Stein CA. Antisense oligonucleotides: basic concepts and mechanisms. Molecular cancer therapeutics. 2002;1(5):347-55.

2. Stein CA, Castanotto D. FDA-Approved Oligonucleotide Therapies in 2017. Molecular therapy : the journal of the American Society of Gene Therapy. 2017;25(5):1069-75.

3. Crooke ST. Molecular Mechanisms of Antisense Oligonucleotides. Nucleic acid therapeutics. 2017;27(2):70-7.

4. Boiziau C, Kurfurst R, Cazenave C, Roig V, Thuong NT, Toulme JJ. Inhibition of translation initiation by antisense oligonucleotides via an RNase-H independent mechanism. Nucleic acids research. 1991;19(5):1113-9.

5. Sazani P, Kole R. Therapeutic potential of antisense oligonucleotides as modulators of alternative splicing. The Journal of clinical investigation. 2003;112(4):481-6.

6. Kolb SJ, Kissel JT. Spinal muscular atrophy: a timely review. Archives of neurology. 2011;68(8):979-84.

7. D'Amico A, Mercuri E, Tiziano FD, Bertini E. Spinal muscular atrophy. Orphanet journal of rare diseases. 2011;6:71.

8. Kashima T, Manley JL. A negative element in SMN2 exon 7 inhibits splicing in spinal muscular atrophy. Nature genetics. 2003;34(4):460-3.

9. Wurster CD, Ludolph AC. Nusinersen for spinal muscular atrophy. Therapeutic advances in neurological disorders. 2018;11:1756285618754459.

10. Finkel RS, Mercuri E, Darras BT, et al. Nusinersen versus Sham Control in Infantile-Onset Spinal Muscular Atrophy. The New England journal of medicine. 2017;377(18):1723-32.

11. Nowak KJ, Davies KE. Duchenne muscular dystrophy and dystrophin: pathogenesis and opportunities for treatment. EMBO reports. 2004;5(9):872-6.

12. Bladen CL, Salgado D, Monges S, et al. The TREAT-NMD DMD Global Database: analysis of more than 7,000 Duchenne muscular dystrophy mutations.
Human mutation. 2015;36(4):395-402.

13. Lim KR, Maruyama R, Yokota T. Eteplirsen in the treatment of Duchenne muscular dystrophy. Drug design, development and therapy. 2017;11:53345.

14. Mendell JR, Rodino-Klapac LR, Sahenk Z, et al. Eteplirsen for the treatment of Duchenne muscular dystrophy. Annals of neurology. 2013;74(5):637-47.

15. Railroading at the FDA. Nature biotechnology. 2016;34(11):1078.

16. ClinicalTrials.gov [Internet]. Bethesda (MD): National Library of Medicine (US). 2000 Feb 29 - . Identifier NCT02255552, Study of Eteplirsen in DMD Patients (PROMOVI); 2014 Oct 2 [cited 2018 July 22]. Available from: https:// clinicaltrials.gov/ct2/show/study/NCT02255552

17. McColgan P, Tabrizi SJ. Huntington's disease: a clinical review. European journal of neurology. 2018;25(1):24-34.

18. Becanovic K, Norremolle A, Neal SJ. A SNP in the HTT promoter alters NFkappaB binding and is a bidirectional genetic modifier of Huntington disease. 2015;18(6):807-16.

19. ClinicalTrials.gov [Internet]. Bethesda (MD): National Library of Medicine (US). 2000 Feb 29 - . Identifier NCT02519036, Safety, Tolerability, Pharmacokinetics, and Pharmacodynamics of IONIS-HTTRx in Patients With Early Manifest Huntington's Disease; 2015 Aug 10 [cited 2018 July 22]. Available from: https://clinicaltrials.gov/ct2/show/study/NCT02519036

20. Rodrigues FB, Wild EJ. Huntington's Disease Clinical Trials Corner: February 2018. Journal of Huntington's disease. 2018;7(1):89-98.

21. Walke DW. IONIS-HTTRX (RG6042) Top-Line Data Demonstrate Significant Reductions of Disease-Causing Mutant Huntingtin Protein in People with Huntington's Disease [Internet]. Carlsbad (CA): Ionis Pharmaceuticals (US); 2018 Mar [cited 2018 July 22]. Available from: https://www.huntingtonsociety.ca/wp-content/uploads/2018/03/2018_03_01_HTT_CHDI_Results_ PR.pdf

22. ClinicalTrials.gov [Internet]. Bethesda (MD): National Library of Medicine (US). 2000 Feb 29 - . Identifier NCT03342053, Study in Huntington's Disease Patients Who Participated in Prior Investigational Studies of ISIS 443139; 2017 Nov 14 [cited 2018 July 22]. Available from: https://clinicaltrials.gov/ ct2/show/NCT03342053

23. Zarei S, Carr K, Reiley L, Diaz K, Guerra O, Altamirano PF, et al. A comprehensive review of amyotrophic lateral sclerosis. Surgical neurology international. 2015;6:171.

24. Miller TM, Pestronk A, David W, Rothstein J, Simpson E, Appel SH, et al. An antisense oligonucleotide against SOD1 delivered intrathecally for patients with SOD1 familial amyotrophic lateral sclerosis: a phase 1, randomised, first-in-man study. The Lancet Neurology. 2013;12(5):435-42.

25. ClinicalTrials.gov [Internet]. Bethesda (MD): National Library of Medicine (US). 2000 Feb 29 - . Identifier NCT02623699, Single and Multiple Dose Study of BlIB067 in Adults With Amyotrophic Lateral Sclerosis (ALS); 2015 Dec 8 [cited 2018 August 8]. Available from: https://www.clinicaltrials.gov/ct2/ show/NCT02623699?term =sod1\%2C+ionis

26. Ando Y, Coelho T, Berk JL, Cruz MW, Ericzon BG, Ikeda S, et al. Guideline of transthyretin-related hereditary amyloidosis for clinicians. Orphanet journal of rare diseases. 2013;8:31.

27. Buxbaum JN. Oligonucleotide Drugs for Transthyretin Amyloidosis. The New England journal of medicine. 2018;379(1):82-5.

28. Benson MD, Waddington-Cruz M, Berk JL, Polydefkis M, Dyck PJ, Wang AK, et al. Inotersen Treatment for Patients with Hereditary Transthyretin Amyloidosis. The New England journal of medicine. 2018;379(1):22-31.

29. The Lancet N. Treating rare disorders: time to act on unfair prices. The Lancet Neurology. 2017;16(10):761.

30. CADTH COMMON DRUG REVIEW - CADTH Canadian Drug Expert Committee Recommendation (Final) [Internet]. Ottawa (ON): CADTH (CA); 2017 Dec [cited 2018 July 22]. Available from: https://www.cadth.ca/sites/default/ files/cdr/complete/SR0525_Spinraza_complete_Dec_22_17.pdf

31. Negishi $Y$, Yamane M, Kurihara N, et al. Enhancement of Blood-Brain Barrier Permeability and Delivery of Antisense Oligonucleotides or Plasmid DNA to the Brain by the Combination of Bubble Liposomes and High-Intensity Focused Ultrasound. Pharmaceutics. 2015;7(3):344-62.

32. Evers MM, Toonen LJ, van Roon-Mom WM. Antisense oligonucleotides in therapy for neurodegenerative disorders. Advanced drug delivery reviews. 2015;87:90-103. 